\title{
Efecto de la poda en la producción de biomasa y proteína en Moringa oleifera Lam. en la zona centro de Veracruz
}

\author{
Pruning effect on biomass and protein production in \\ Moringa oleifera Lam. in the central area of Veracruz
}

\begin{abstract}
Rafael Ruiz-Hernández ${ }^{1}$, Arturo Pérez-Vázquez ${ }^{1 *}$, Cesareo Landeros-Sánchez¹, Ofelia Andrea Valdes Rodríguez² Katia Angélica Figueroa-Rodríguez ${ }^{3}$

Colegio de Postgraduados, Campus Veracruz. Carretera federal Xalapa-Veracruz km 88.5, CP 91700 México.

El Colegio de Veracruz. Carrillo Puerto No. 26, zona centro. CP 91000 . Xalapa, Veracruz, México.

3 Colegio de Postgraduados, Campus Córdoba. Carretera Federal Córdoba-Veracruz, Km 348, Congregación Manuel León Amatlán de los Reyes, Veracruz, C.P. 94500. México.
\end{abstract}

\section{RESUMEN}

Moringa oleifera es una especie con gran importancia alimenticia humana y animal. El objetivo fue determinar el efecto de la poda sobre la producción de biomasa y contenido proteico en moringa. Plantas de tres años de edad fueron seleccionadas de acuerdo a la similitud entre altura y diámetro basal. El diseño experimental fue en bloques completamente al azar con tres tratamientos y cuatro repeticiones. Los tratamientos fueron podas de tallo a 0.75 (T1), 1.00 (T2) y $1.50 \mathrm{~m}$ (T3) de altura. Se midieron variables de longitud de brotes, número de brotes, número de hojas y producción de biomasa a los cuatro meses. Paralelamente se determinó el contenido de humedad, ceniza y proteína en hojas. Se encontraron diferencias estadísticas (Tukey, $\mathrm{p}<0.05$ ) en el crecimiento y supervivencia de los brotes, siendo los tratamientos $1.00 \mathrm{~m}$ y $0.75 \mathrm{~m}$ de mayor altura para la primera y segunda poda. El T3 presentó $83.31 \%$ de humedad, 16.85 \% de proteína y $12.3 \%$ de ceniza en la primera poda. No se encontraron diferencias estadísticas (Tukey, p>0.05) en la producción de biomasa, ni tampoco en el contenido de proteína en hojas. Se concluye que, moringa tolera podas a baja altura. La práctica de las podas incrementa la producción de biomasa y facilitan la cosecha en moringa.

Palabras clave: producción, cosecha.

\section{SUMMARY}

Moringa oleifera is a species with great nutritional importance. The objective of this work was to determine the effect of pruning in moringa biomass production and protein content. Three-year-old plants were selected according to the similarity between height and basal diameter. The experimental design consisted on three treatments and four repetitions. The treatments were pruned at 0.75 (T1), 1.00 (T2) and $1.50 \mathrm{~m}$ (T3) in height. The variables measured were shoot length, number of shoots, number of leaves and biomass production at four months. In parallel, moisture, ash and protein content in leaves were determined. Statistical differences (Tukey, $\mathrm{p}<0.05$ ) were found in the shoots growth and survival, being the treatments $1.00 \mathrm{~m}$ and $0.75 \mathrm{~m}$ of grea- ter height for the first and second pruning. The T3 presented $83.31 \%$ humidity, $16.85 \%$ protein and $12.3 \%$ ash in the first pruning. No statistical differences (Tukey, $p>0.05$ ) were found in biomass production, nor in leaf protein content. It is concluded that moringa is tolerant to pruning at low height. Pruning, as agricultural practice, increases biomass production and facilitates harvesting in moringa.

Keywords: Moringa, production, harvest.

\section{INTRODUCCIÓN}

Ante los retos actuales de la seguridad alimentaria, perdida de la fertilidad de suelos y cambio climático, se busca que mediante la agricultura se pueda mitigar el hambre y la desnutrición que sufre la humanidad (FAO, 2017). Una alternativa que puede contribuir a esta noble tarea es el cultivo de moringa (Moringa oleifera Lam.), un árbol multipropósito originario del Noroeste de la India que se ha distribuido en la mayoría de las zonas tropicales y subtropicales del mundo y que se introdujo a América Latina en el año de 1920 (Olson y Fahey, 2011).

Moringa presenta una diversidad de usos, entre ellos: industrial, floculante, bioabsorbente, agrícola, medicinal y alimenticio, siendo estos dos últimos los de mayor interés mundial (Anwar, 2007; Madera-Santana et al., 2019). Las hojas contienen prácticamente todos los aminoácidos esenciales, carbohidratos, carotenos, vitaminas, calcio, hierro, magnesio, fósforo, potasio, zinc, proteínas, grasas y ácidos grasos (CheIliah et al., 2017). Éstas se utilizan en la alimentación tanto humana como animal promoviendo muchos beneficios productivos y un mejor estado nutricional (Kakengi et al., 2007).

Pocas plantas en el mundo se comparan con el perfil nutricional de moringa, además presenta un rápido crecimiento, alta producción de biomasa, supervivencia en climas adversos, alta tolerancia a la sequía, crece adecuadamente en precipitaciones de 500 a 1,500 mm, suelos francos o delgados, con poca cantidad de nutrientes y con intervalo de $\mathrm{pH}$ de 4.5-8.0 (Adedapo et al., 2009). Es una planta que puede producir una alta cantidad de biomasa (fresca y seca) aún a altas densidades de siembra (Reyes et al., 2006). Sin 
embargo, la respuesta a podas cuando el diámetro del tallo es pequeño $(5-10 \mathrm{~mm}$ ) limita la producción de ramas (Foidl et al., 2011). Moringa se puede utilizar como una opción sostenible y económicamente viable para la producción de alimentos, reforestación, producción de forraje o cultivo agrícola resistente al cambio climático (Reyes et al., 2006; Gandji et al., 2018). También puede usarse como un recurso clave en la alimetación para combatir la desnutrición, obesidad y patologías asociadas a la carencia de vitaminas (Orona et al., 2015; Rodiles-López et al., 2019). El uso de moringa con fines nutricionales puede contribuir a la seguridad alimentaria y nutricional de la población, principalmente en comunidades rurales donde el acceso a alimentos y su calidad nutriconal es inadecuado (Mudyiwa et al., 2013; Aguilar-Jiménez et al., 2019).

En el sureste de México existen hoy día una gran cantidad de unidades de producción comercial de moringa, las cuales desconocen las mejores prácticas de manejo agronómico como la mejor altura de poda, frecuencia de corte y densidad de siembra para obtener una mayor cantidad y calidad en la producción de biomasa. Se sabe, que el rendimiento de biomasa fresca y seca en moringa esta determinado por las condiciones edafoclimáticas y prácticas de manejo del cultivo (Ramos et al., 2015). Moringa responde muy bien a podas con una alta capacidad de rebrote y permite tener varias cosechas de hoja al año. Pero a medida que la planta envejece disminuye su producción (Palada y Chang, 2003). Una de las estrategias para aumentar su producción de biomasa es la poda (Ledea et al., 2018).

La poda de tallo promueve la ramificación, aumenta el rendimiento y en alturas adecuadas facilita la cosecha. Cabe mencionar que existe poca información sobre el efecto de la poda en la producción de biomasa y contenido de proteína. Por ello, se requiere de investigaciones que determinen la altura óptima de corte para que la planta logre una producción de biomasa sin poner en riesgo la supervivencia del cultivo. Por tanto, el objetivo fue determinar el efecto de la poda sobre la producción de biomasa y contenido de proteína en moringa en la zona centro del estado de Veracruz. Bajo la hipótesis de que la producción de biomasa y contenido de proteína de moringa esta en función con la altura de poda.

\section{MATERIALES Y MÉTODOS}

\section{Área de estudio y material biológico}

La unidad experimental se estableció en los terrenos del Colegio de Postgraduados, Campus Veracruz, localizado en el municipio de Manlio Fabio Altamirano $\left(19^{\circ} 16^{\prime} 00^{\prime \prime} \mathrm{LN}\right.$, $96^{\circ} 16^{\prime} 32^{\prime \prime}$ LO; 16 msnm). La zona esta caracterizada por tener un clima trópico subhúmedo con lluvias en verano durante cuatro meses, del mes de junio a septiembre. Durante el experimento se registraron datos climáticos. El promedio de temperatura máxima fue de $31^{\circ} \mathrm{C}( \pm 1.94)$ y mínima de 22 ${ }^{\circ} \mathrm{C}( \pm 1.21)$; la humedad relativa de $82 \%( \pm 2.11)$ y una precipitación acumulada de $293 \mathrm{~mm}$ y la anual de ese año de $771.2 \mathrm{~mm}$. Se realizó un perfil de suelo a una profundidad de $1.27 \mathrm{~m}$ para identificar las capas del suelo y describir las características de cada capa. El suelo se caracteriza ser poco profundo y pedregoso. Se realizó el análisis de suelo a cada capa del perfil y se determinó el color, \% de materia orgánica, pedregosidad, textura, densidad aparente, biodiversidad, conductividad eléctrica $(\mu \mathrm{S}), \mathrm{pH}$, amoníaco $\left(\mathrm{NH}_{3}-\mathrm{N}\right), \mathrm{P}, \mathrm{K}, \mathrm{y}$ nitrato $\left(\mathrm{NO}_{3}-\mathrm{N}\right)$, bajo la NOM-021-SEMARNAT-2000 (SEMARNAT, 2002).

Las semillas de moringa fueron sembradas en octubre del 2012, cuyo peso promedio fluctuaba en 268.9 ( \pm 42.9) mg. Para julio del 2015, los árboles del cultivo tenían una edad de 3 años.

\section{Tratamientos y Diseño experimental}

El diseño fue de bloques completamente al azar con tres bloques, tres tratamientos y cuatro repeticiones por bloque (Tabla 1). Los tratamientos se determinaron seleccionando aquellas plantas que tuvieran altura y diámetro del tallo similar. La altura promedio de las plantas fue de $6.41( \pm$ 0.22) $\mathrm{m}$ y un diámetro promedio del tallo de 13.78 ( \pm 1.69 ) $\mathrm{cm}$. La distancia entre filas fue de $4 \mathrm{~m}$ y $2 \mathrm{~m}$ entre plantas. La poda se realizó con serrotes a los tratamientos de altura de $0.75,1.00,1.50 \mathrm{~m}$. La primera poda se realizó el día 27 de julio y la cosecha el 28 de septiembre ( 63 días después de la poda), y la segunda cosecha el 28 de noviembre (61 días después de la poda).

Tabla 1. Tratamientos de altura de poda en el cultivo de M. oleifera. Table 1. Pruning height treatments in the cultivation of $M$. oleifera.

\begin{tabular}{lc}
\hline Tratamiento & Altura de poda respecto al suelo \\
\hline T1 & $0.75 \mathrm{~m}$ \\
T2 & $1.00 \mathrm{~m}$ \\
T3 & $1.50 \mathrm{~m}$ \\
\hline
\end{tabular}

\section{Manejo del experimento y variables registradas}

Diariamente se monitoreaba las plantas para evitar que fueran perjudicadas por algún agente externos como plagas o enfermedades. Las plantas no recibieron riego ni abono durante la experimentación.

Cada siete días se registraron las variables: altura, número de ramas y hojas de los brotes. Con ayuda de un flexómetro se midió la longitud de los rebrotes. Se realizaron dos muestreos con periodos de 63 y 61 días para conocer la producción de biomasa fracción gruesa (brotes) y fracción fina (hojas). Se determinó la tasa de crecimiento promedio en los tratamientos. El contenido de materia seca se determinó deshidratando el tallo y las hojas (fracción fina y gruesa) en una estufa de aire forzado a una temperatura de $60{ }^{\circ} \mathrm{C}$ durante $48 \mathrm{~h}$. Se utilizaron hojas deshidratadas previamente molidas para determinar la cantidad de humedad, ceniza y proteína. Para la determinación del contenido de proteína su utilizó el Método de Kjeldahl (AOAC, 1984) y para el contenido de ceniza se utilizó el método establecido en la NMX-F066-S-1978 (DOF, 1961). 


\section{Análisis estadístico}

Para determinar diferencias estadísticas entre las diferentes alturas de poda se realizaron análisis de varianza con la prueba de Tukey $(\mathrm{p} \leq 0.05)$ por cada variable registrada. El análisis estadístico y las correlaciones se realizaron con el programa InfoStat versión 2016. Se estimó el promedio de los tratamientos contemplando el diseño experimental.

\section{RESULTADOS}

\section{Perfil del suelo}

En la capa superior del suelo $(0-0.25 \mathrm{~m})$ se encontró la mayor cantidad de materia orgánica y un suelo más ligero, y a mayor profundidad se identificó mayor compactación (Tabla 2).

Tabla 2. Carcateristicas fisicoquímicas del perfil de suelo en el área experimental donde se establecieron las plantas de $M$. oleifera.

Table 2. Physicochemical characteristics of the soil profile in the experimental area where the $M$. oleifera plants were established.

\begin{tabular}{|c|c|c|}
\hline $\begin{array}{l}\text { Profundidad } \\
(\mathrm{cm})\end{array}$ & Carácter & Descripción \\
\hline \multirow{8}{*}{$0-25$} & Color & Amarillo grisáceo en húmedo \\
\hline & Materia orgánica & $2.5 \%$ \\
\hline & Raíces & Gruesas medianas \\
\hline & Pedregosidad & Tamaño pequeño \\
\hline & Textura & Franco arcillosa \\
\hline & Densidad aparente & Buena $1.2-1.27$ \\
\hline & Biodiversidad & Hormigas y microfauna \\
\hline & Observaciones & $\begin{array}{c}\text { Presencia de manchas } \\
\text { moteadas }\end{array}$ \\
\hline \multirow{7}{*}{$25.1-53$} & Color & Café cobrizo en húmedo \\
\hline & Materia orgánica & Mínima cantidad de M.O \\
\hline & Raíces & $\begin{array}{l}\text { Tope de desarrollo radicular } \\
\text { por la presencia de piedra }\end{array}$ \\
\hline & Pedregosidad & $\begin{array}{c}\text { Abundante con capas } \\
\text { laminares con segmentos } \\
\text { duros }\end{array}$ \\
\hline & Textura & Franco-arcilloarenosa \\
\hline & Densidad aparente & $1.3-1.4$ \\
\hline & Diámetro de piedra & Superior a los $8 \mathrm{~cm}$ \\
\hline \multirow{4}{*}{$53.1-87$} & Color & Amarillo grisáceo en húmedo \\
\hline & Materia orgánica & Ausente \\
\hline & Gravilla & $\begin{array}{l}\text { Producto del desgaste del } \\
\text { material rocoso de la capa } \\
\text { superior }\end{array}$ \\
\hline & Moteados & $\begin{array}{l}\text { Color amarillo y material } \\
\text { laminar duro }\end{array}$ \\
\hline \multirow{5}{*}{$87.1-127$} & Color & Gris claro \\
\hline & Materia orgánica & Ausente \\
\hline & Roca madre & Sin presencia de piedra \\
\hline & Raíces & Escasas raíces adventicias \\
\hline & Pedregosidad & Material laminar compactado \\
\hline
\end{tabular}

\section{Análisis de suelo}

El análisis de suelo arrojó que la capa superior (0 - 0.25 m) presentó mayores valores de conductividad eléctrica y materia orgánica en comparación con las capas de mayor profundidad. El valor de $\mathrm{pH}$ fue ligeramente ácido en la superficie y neutro a mayor profundidad, con un nivel medio de materia orgánica en los primeros $25 \mathrm{~cm}$ y menor contenido a mayor profundidad. El contenido de $\mathrm{N}$ fue $2.5 \mathrm{mg} \mathrm{L}^{-1}, \mathrm{P} 1$ $\mathrm{mg} \mathrm{L}^{-1}$ y K $14 \mathrm{mg} \mathrm{L}^{-1}$ en la capa superficial (Tabla 3). La textura franco arcillosa en la capa superficial y arenosa-franca a una profundidad mayor a los $25 \mathrm{~cm}$. Por tanto, puede ser ubicado como un suelo con buen drenaje y apto para el cultivo de moringa.

La primera y segunda poda se realizaron el día 27 de julio y 28 de septiembre, respectivamente. El efecto de la poda se reflejó en la aparición de los primeros rebrotes, a los 20 y 21 días después de la primera y segunda poda. La tasa de crecimiento relativo (TCR) tuvo un decremento respecto a la altura de poda. La TCR para las alturas $0.75,1.00$ y $1.50 \mathrm{~m}$ fueron de 1.30, 1.35 y $1.10 \mathrm{~cm} \mathrm{día}^{-1}$, siendo el tratamiento de $1.00 \mathrm{~m}$ el de mayor crecimiento en la primera poda realizada a los 63 días. Durante los primeros 28 días, el tratamiento de 1.00 mostró diferencias estadisticas $(p<0.05)$ en el crecimiento de altura respecto a los otros tratamientos.

En la segunda poda, las TCR fueron de 1.80, 1.44 y 0.98 $\mathrm{cm}$ día $^{-1}$ para las alturas $0.75,1.00$ y $1.50 \mathrm{~m}$. En este caso el tratamiento con mayor tasa de crecimiento fue a $0.75 \mathrm{~m}$. Los tratamientos 0.75 y $1.00 \mathrm{~m}$ mostraron diferencias significativas $(p<0.05)$ en el crecimiento desde los 26 días después de la segunda poda, siendo similar al final del periodo de experimentación. La Figura 1 muestra las curvas de crecimiento promedio de los tratamientos durante dos periodos (63 y 61) de poda y cosecha. En el primer periodo, el mayor crecimiento correspondió al tratamiento de $1.00 \mathrm{~m}$ y en el segundo periodo fue el tratamiento de $0.75 \mathrm{~m}$.

\section{Número de hojas}

En el primer periodo existió una diferencia significativa $(p<0.05)$ en el número de hojas entre T1 $(0.75 \mathrm{~m})$ respecto a los otros tratamientos; para el día 60 , la altura de T3 $(1.50 \mathrm{~m})$ fue la más productiva en el número de hojas. En el segundo periodo, durante los primeros 26 días después de la poda no se encontró diferencias significativas entre tratamientos; el incremento de hojas para la altura $1.50 \mathrm{~m}$ fue evidente a partir del día 40 diferenciándose de los otros tratamientos. Mientras que la poda a 1.00 y $1.50 \mathrm{~m}$ presentaron un número de hojas similar. El T2 $(1.00 \mathrm{~m})$ fue el más productivo en el primer periodo y el T3 $(1.50 \mathrm{~m})$ en el segundo periodo (Figura 2).

\section{Producción de brotes}

Los brotes emergieron a los 20 días después de la poda, pero no todos llegaron a desarrollarse por completo. En el primer periodo los tratamientos 1.00 y $1.50 \mathrm{~m}$ tuvieron cantidades similares de brotes durante los primeros 53 días, a partir de ahí se presentó un incremento significativo de brotes en el T3 $(1.50 \mathrm{~m})$ respecto a los otros tratamientos. 
Tabla 3. Análisis de suelo a las diferentes profundidades del área de cultivo de M. oleifera.

Table 3. Soil analysis at different depths of the M. oleifera cultivation area.

\begin{tabular}{lcccccccc}
\hline $\begin{array}{l}\text { Profundidad } \\
(\mathbf{c m})\end{array}$ & Textura & $\begin{array}{c}\text { Conductividad } \\
\text { eléctrica }(\mu \mathrm{S})\end{array}$ & $\mathbf{p H}$ & $\begin{array}{c}\text { Materia } \\
\text { orgánica } \\
(\%)\end{array}$ & $\begin{array}{c}\text { Amoniaco } \\
\mathbf{N H}_{\mathbf{3}}-\mathbf{N} \\
\left(\mathbf{m g ~ L}^{-1}\right)\end{array}$ & $\begin{array}{c}\mathbf{P} \\
\left(\mathbf{m g ~ L}^{-1}\right)\end{array}$ & $\begin{array}{c}\mathbf{K} \\
\left(\mathbf{m g ~ L}^{-1}\right)\end{array}$ & $\begin{array}{c}\text { Nitrato } \\
\left(\mathbf{N O} \mathbf{s}_{\mathbf{3}} \mathbf{N}\right) \\
\left(\mathbf{m g ~ L}^{-1}\right)\end{array}$ \\
\hline $0-25$ & $\begin{array}{c}\text { Franco } \\
\text { arcilloso }\end{array}$ & 186.8 & 6.34 & 2.50 & 2.5 & 1 & 14 & 86 \\
\hline $25.1-53$ & $\begin{array}{c}\text { Arenoso } \\
\text { Franco }\end{array}$ & 145.0 & 6.87 & 0.48 & 2.0 & 1.5 & 24 & 12 \\
\hline $53.1-87$ & $\begin{array}{c}\text { Arenoso } \\
\text { Franco }\end{array}$ & 114.6 & 6.77 & 0.36 & - & - & - & 10 \\
\hline $87.1-127$ & $\begin{array}{c}\text { Franco } \\
\text { arcilloso }\end{array}$ & 218.1 & 6.91 & 0.30 & - & - & - & - \\
\hline
\end{tabular}
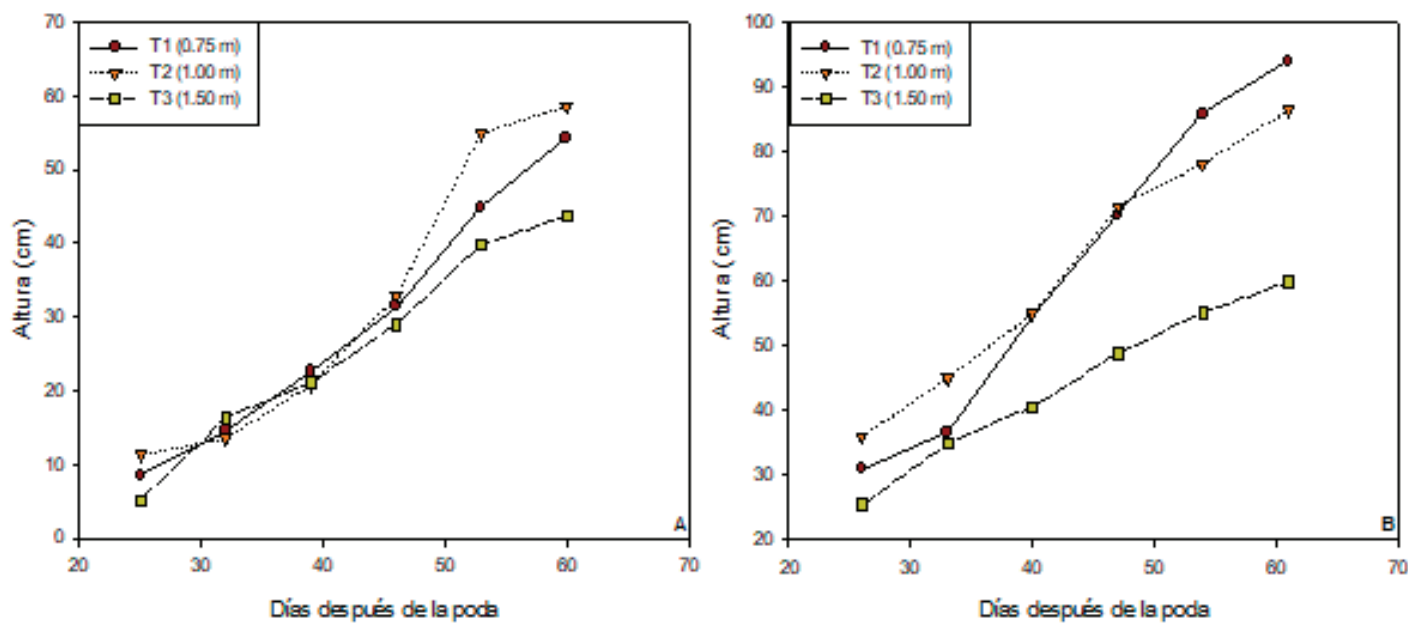

Figura 1. Crecimiento promedio de brotes de M. oleifera a diferentes alturas de poda en dos periodos: A) Periodo 1: altura máxima T2: $58 \mathrm{~cm}$; y B) Periodo 2: altura máxima T1: $93 \mathrm{~cm}$.

Figure 1. Average growth of $M$. oleifera shoots at different pruning heights at two periods: A) Period 1: maximum height T2: $58 \mathrm{~cm}$; and B) Period 2: maximum height $\mathrm{T} 1: 93 \mathrm{~cm}$.
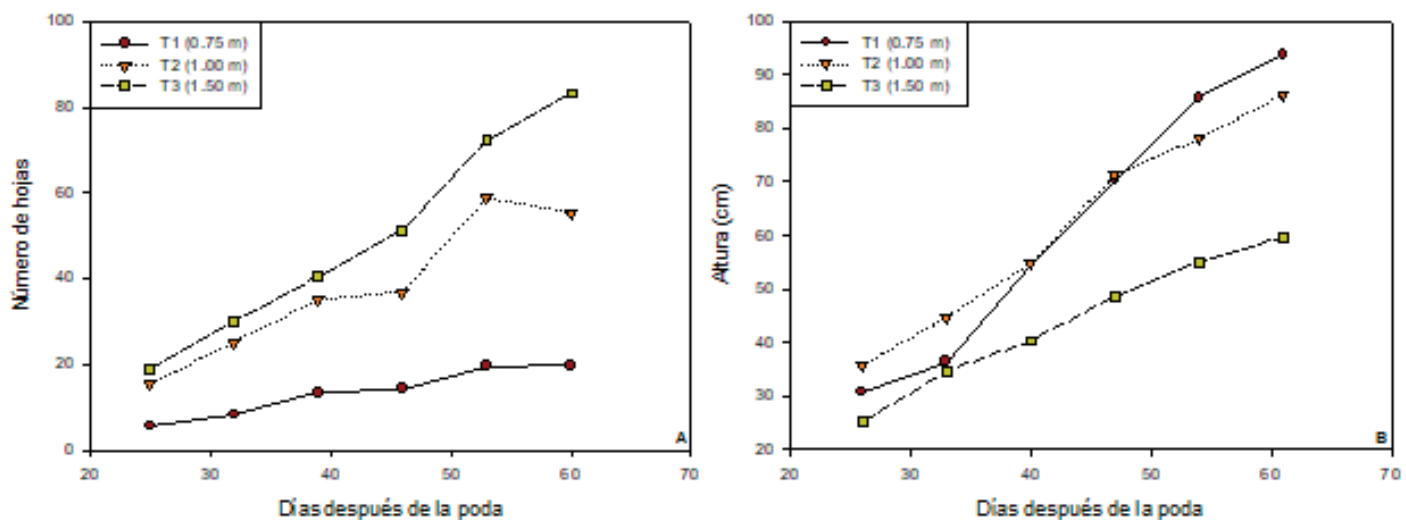

Figura 2. Número de hojas de M. oleifera a diferentes alturas de poda en dos periodos de poda A) Periodo 1: tratamiento con mayor cantidad de hojas T3: 83 hojas, B) Periodo 2: tratamiento con mayor cantidad de hojas, T3:115 hojas.

Figure 2. Number of $M$. oleifera leaves at different pruning heights at two pruning periods $A$ ) Period 1: treatment with a greater quantity of leaves T3: 83 leaves, B) Period 2: treatment with a greater quantity of leaves, T3: 115 sheets. 
En el segundo periodo se encontró una diferencia significativa entre el T3 respecto a los otros tratamientos (Figura 3). El número máximo de rebrotes correspondió a la altura de poda de $1.50 \mathrm{~m}$ con 10 brotes y el menor correspondió a 0.5 m con 4. La mayor desviación estándar (4.72) en la producción de rebrotes correspondió al $1.50 \mathrm{~m}$, y la menor a 0.75 $\mathrm{m}$ (2.36). Aunque el mayor número de brotes correspondió a una altura de $1.50 \mathrm{~m}$, esto se relacionó con la producción de biomasa, debido al tamaño de los rebrotes y hojas.

\section{Producción de biomasa}

Respecto a la producción de biomasa no se encontraron diferencias estadísticas $(p>0.05)$ en ambas cosechas. Se encontró una tendencia a que a una altura de poda de 1.00 $\mathrm{m}$ tuvo una mayor producción de biomasa (fracción gruesa y fina) para el primer periodo, dando un rendimiento equivalente a $0.24 \mathrm{t} \mathrm{ha}^{-1}$ de materia seca superior a los 0.030 y 0.055 $\mathrm{t} \mathrm{ha}^{-1}$ para los $0.75 \mathrm{~m}$ y $1.00 \mathrm{~m}$. En el segundo periodo, el T3 tuvo la mayor producción de biomasa fracción fina seca. El resultado demuestra que el T1 $(0.75 \mathrm{~m})$ tuvo una producción baja (Tabla 4).

\section{Análisis bromatológico}

No se encontraron diferencias significativas respecto a la cantidad de humedad, proteína y ceniza contenida en las hojas en ambos periodos (Tabla 5). La cantidad de proteína y de ceniza en la primera cosecha fue de $16.48 \%$ y $11.78 \%$ y en la segunda, el contenido de proteína y de ceniza fue de $18.52 \%$ y el $11.33 \%$. Los contenidos de humedad en la hoja fueron de 79 y $76 \%$ en la primera y segunda cosecha. Comparando entre cosechas, se encontró que los valores más altos, de proteína y ceniza y menor contenido de humedad en la segunda poda. La cantidad de humedad en la hoja estuvo influenciada por la precipitación de $92.2 \mathrm{~mm}$ inferior a los $198.6 \mathrm{~mm}$ registrados en el primer periodo.

En la primera poda, la correlación de la altura de poda con la cantidad de proteína (0.17) y ceniza fue baja (0.45). Se encontró una correlación positiva entre la altura del brote y el peso del tallo (0.79) y hoja (0.70). La altura de poda tuvo una correlacion de 0.47 con la TCR de la hoja (Tabla 6).

En la segunda poda, la correlación de la altura de poda con la cantidad de proteína $(-0.27)$ y ceniza fue negativa $(-0.22)$. Hubo una correlación positiva entre la altura de poda
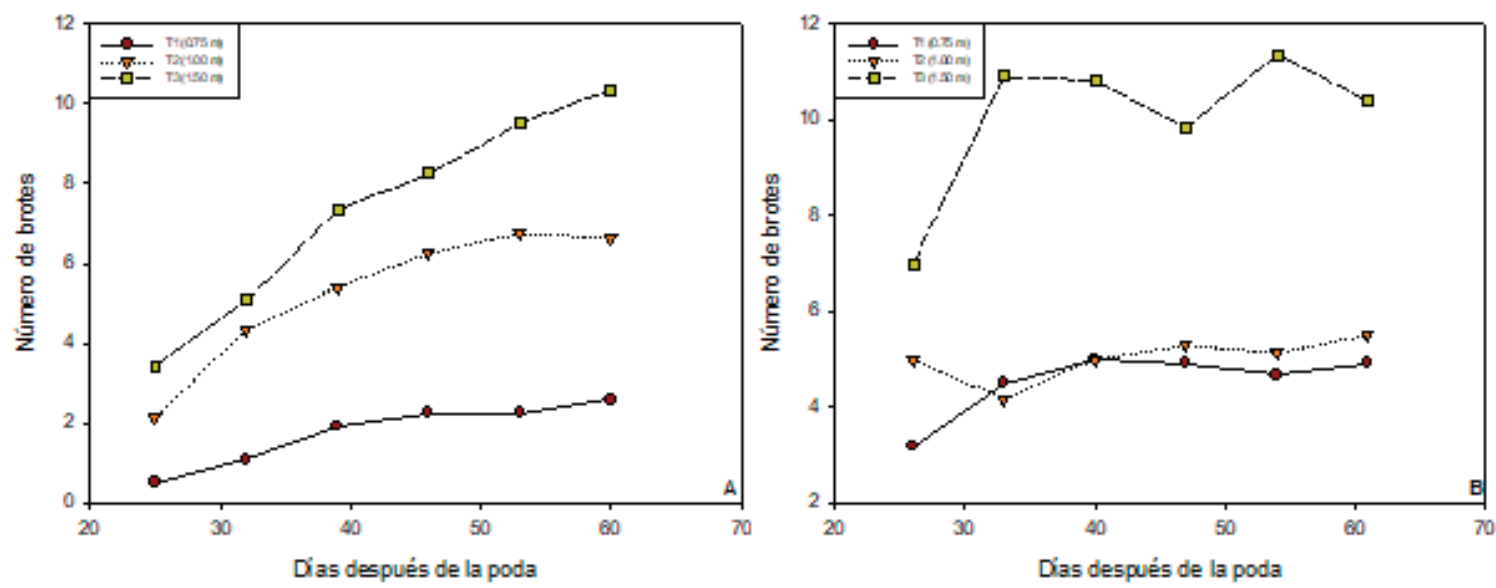

Figura 3. Número de brotes de M. oleifera a diferentes alturas de poda en dos periodos de poda A) Periodo 1:63 días B) Periodo 2: 61 días.

Figure 3. Number of shoots of M. oleifera at different pruning heights at two pruning periods A) Period 1:63 days B) Period 2:61 days.

Tabla 4. Producción de biomasa de M. oleifera a diferentes alturas de poda en dos periodos de poda. Table 4. Biomass production of $M$. oleifera at different pruning heights in two pruning periods.

\begin{tabular}{lccccc}
\hline Poda & $\begin{array}{c}\text { Tratamiento (altura } \\
\text { de poda) }\end{array}$ & $\begin{array}{c}\text { Biomasa F. F. } \\
\text { (fresco) (g) }\end{array}$ & $\begin{array}{c}\text { Biomasa F. G. } \\
\text { (fresco) (g) }\end{array}$ & $\begin{array}{c}\text { Biomasa F. F. (seco) } \\
\text { (g) }\end{array}$ & $\begin{array}{c}\text { Biomasa F. G. (seco) } \\
\text { (g) }\end{array}$ \\
\hline 1ra & T1 $(0.75 \mathrm{~m})$ & $130.53^{\mathrm{a}}$ & $133.50^{\mathrm{a}}$ & $22.99^{\mathrm{a}}$ & $23.27^{\mathrm{a}}$ \\
& T2 $(1.00 \mathrm{~m})$ & $314.30^{\mathrm{a}}$ & $315.97^{\mathrm{a}}$ & $75.63^{\mathrm{a}}$ & $74.75^{\mathrm{a}}$ \\
& T3 $(1.50 \mathrm{~m})$ & $270.70^{\mathrm{a}}$ & $225.51^{\mathrm{a}}$ & $41.81^{\mathrm{a}}$ & $39.17^{\mathrm{a}}$ \\
\hline \multirow{2}{*}{$\mathrm{ra}$} & T1 $(0.75 \mathrm{~m})$ & $648.34^{\mathrm{a}}$ & $817.84^{\mathrm{a}}$ & $150.35^{\mathrm{a}}$ & $128.16^{\mathrm{a}}$ \\
& T2 $(1.00 \mathrm{~m})$ & $628.34^{\mathrm{a}}$ & $716.69^{\mathrm{a}}$ & $135.43^{\mathrm{a}}$ & $92.80^{\mathrm{a}}$ \\
& T3 $(1.50 \mathrm{~m})$ & $677.28^{\mathrm{a}}$ & $697.51^{\mathrm{a}}$ & $137.17^{\mathrm{a}}$ & $111.54^{\mathrm{a}}$ \\
\hline
\end{tabular}

*Valor promedio de tres repeticiones. FF (fración fina); FG (fración gruesa) 
Tabla 5. Contenido de humedad, proteína y ceniza de M. oleifera a diferentes alturas de poda.

Table 5. Moisture, protein and ash content of M. oleifera at different pruning heights.

\begin{tabular}{lcccccc}
\hline \multirow{2}{*}{ Tratamiento } & \multicolumn{3}{c}{ 1ra poda } & \multicolumn{3}{c}{ 2ra poda } \\
\cline { 2 - 7 } & Humedad (\%) & $\begin{array}{c}\text { Proteína base } \\
\text { seca (\%) }\end{array}$ & Ceniza (\%) & Humedad (\%) & $\begin{array}{c}\text { Proteína base } \\
\text { seca (\%) }\end{array}$ & Ceniza (\%) \\
\hline T1 $(0.75 \mathrm{~m})$ & 76.83 & 16.37 & 10.6 & 75.62 & 18.6 & 11.3 \\
T2 $(1.00 \mathrm{~m})$ & 79.50 & 16.22 & 12.3 & 77.17 & 19.9 & 11.6 \\
\hline T3 $(1.50 \mathrm{~m})$ & 83.81 & 16.85 & 12.3 & 77.44 & 17.7 & 11.0 \\
\hline
\end{tabular}

Tabla 6. Correlaciones entre las variables registradas en la producción de biomasa de $M$. oleifera a diferentes alturas de poda de la primera cosecha. Table 6. Correlations between the recorded variables in the $M$. oleifera biomass production at different pruning heights of the first harvest.

\begin{tabular}{|c|c|c|c|c|c|c|c|c|c|c|c|}
\hline & Tratamiento & $\begin{array}{l}\text { Altura } \\
\text { del brote }\end{array}$ & Ramas & Hojas & $\begin{array}{c}\% \\
\text { Materia } \\
\text { seca }\end{array}$ & $\begin{array}{c}\% \\
\text { Ceniza }\end{array}$ & $\begin{array}{c}\% \\
\text { Proteina }\end{array}$ & $\begin{array}{l}\text { Peso } \\
\text { F. hoja }\end{array}$ & $\begin{array}{l}\text { Peso } \\
\text { F. tallo }\end{array}$ & $\begin{array}{c}\text { TCR } \\
\text { HOJA }\end{array}$ & $\begin{array}{c}\text { TCR } \\
\text { TALLO }\end{array}$ \\
\hline Tratamiento & 1 & & & & & & & & & & \\
\hline Altura del brote & -0.18 & 1 & & & & & & & & & \\
\hline Ramas & 0.93 & -0.07 & 1 & & & & & & & & \\
\hline Hojas & 0.88 & 0.23 & 0.95 & 1 & & & & & & & \\
\hline Materia seca & 0.48 & 0.38 & 0.55 & 0.66 & 1 & & & & & & \\
\hline$\%$ Ceniza & 0.45 & 0.54 & 0.61 & 0.77 & 0.73 & 1 & & & & & \\
\hline$\%$ Proteina & 0.17 & 0.30 & 0.15 & 0.22 & -0.29 & -0.16 & 1 & & & & \\
\hline Peso F. hoja & 0.24 & 0.70 & 0.44 & 0.62 & 0.54 & 0.73 & 0.26 & 1 & & & \\
\hline Peso F. tallo & 0.14 & 0.79 & 0.31 & 0.54 & 0.49 & 0.73 & 0.23 & 0.98 & 1 & & \\
\hline TCR ${ }^{*}$ HOJA & 0.47 & 0.57 & 0.66 & 0.81 & 0.68 & 0.92 & 0.04 & 0.9 & 0.86 & 1 & \\
\hline TCR TALLO & 0.42 & 0.6 & 0.59 & 0.76 & 0.7 & 0.95 & -0.05 & 0.88 & 0.88 & 0.99 & 1 \\
\hline
\end{tabular}

y el número de ramas (0.81) y hoja (0.76). La altura de poda tuvo una correlacion de -0.41 con la TCR de la hoja (Tabla 7).

En la Figura 4 se muestra la regresión de la altura con el peso de la hoja seca.

\section{DISCUSIÓN}

Los arboles de moringa del experiento tenían 3 años de edad, con crecimiento monopódico, con una o dos ramas, en un suelo franco-arcilloso y sin fertilización. Debido a la compactación del suelo, el desarrollo de la raíz se vió limitado, alcanzando una profundidad de $60 \mathrm{~cm}$ y en algunos casos con bifurcación.

La poda es una práctica agronómica que incrementa la ramificación, la producción de hojas y facilita la cosecha (Leone et al., 2015). La poda es importante para la producción de nuevos rebrotes eliminando la dominancia apical (Crosby y Craker, 2007). Esta práctica evita que las hojas y los frutos se produzcan en el tallo principal (Palada y Chang, 2003). Además, tiene un efecto positivo en el diámetro, número de inflorescencias, número y tamaño de los frutos debido al mayor desarrollo de dosel (Du Toit et al., 2019). La utilización de la poda en épocas claves del año permite la producción de más flores y frutos fuera de temporada lo cual favorece a los productores (Sharmila et al., 2018). Por ello, se recomienda realizar la poda cuando el árbol rebase $1 \mathrm{~m}$ de altura ó cuando el tallo sea demasiado grueso (Noamesi et al., 2010). Para fomentar la ramificación se puede podar a 1 o $2 \mathrm{~m}$ de la atura o recortar las ramas a $30 \mathrm{~cm}$ por arriba de un nudo (Palada y Chang, 2003). Montesinos (2010) sugiere podar a $20 \mathrm{~cm}$ del suelo para lograr mayor producción de biomasa y Lawal et al. (2015) mencionan que puede podarse de 0.40 a $1.50 \mathrm{~m}$ debido a la gran capacidad de producción de rebrotes (López et al., 2012). En este estudio se encontró un rápido crecimiento y una alta supervivencia de los rebrotes. La capacidad de rebrote se debe a la traslocacion de fotosintatos de la raíz a la parte superior del tallo, permitiendo el desarrollo de brotes, hojas y un incremento en el rendimiento de materia seca (Nouman et al., 2013). Además, favorece el desarrollo de la raíz e incrementa la eficiencia de los agroquímicos como fertilizantes (Strik y Buller, 2005), puede rejuveneserce la planta y mantenerla a una altura que permita la cosecha de hojas sin problemas o riesgos.

Las condiciones del clima, las cuales fueron propicias, permitieron obtener buenos resultados al realizar podas en periodos de 63 y 61 días. Se encontró que la altura óptima de poda es a $1.00 \mathrm{~m}$ de altura con un diámetro de tallo de 137.84 $( \pm 16.92) \mathrm{mm}$, logrando buena producción de biomasa en un periodo de 63 días. La producción de biomasa en moringa se vió afectada por el nivel de precipitación durante el periodo de experimentación, siendo un año con escasa precipitación respecto al promedio anual para la zona (1,100 mm). Padilla et al. (2017) sugieren que las podas deben realizarse en épocas de lluvias y no en verano (estiaje). Las podas en épocas cálidas ocasionan que los brotes sean más cortos y 
Tabla 7. Correlaciones entre las variables de la producción de biomasa de $M$. oleifera a diferentes alturas de poda de la segunda cosecha. Table 7. Correlations between the biomass production variables of $M$. oleifera at different pruning heights of the second harvest.

\begin{tabular}{|c|c|c|c|c|c|c|c|c|c|c|c|}
\hline & Tratamiento & $\begin{array}{l}\text { Altura } \\
\text { del brote }\end{array}$ & Ramas & Hojas & $\begin{array}{c}\% \\
\text { Materia } \\
\text { seca }\end{array}$ & $\begin{array}{c}\% \\
\text { Ceniza }\end{array}$ & $\begin{array}{c}\% \\
\text { Proteina }\end{array}$ & $\begin{array}{l}\text { Peso } \\
\text { F. hoja }\end{array}$ & $\begin{array}{c}\text { Peso } \\
\text { F. tallo }\end{array}$ & $\begin{array}{c}\text { TCR } \\
\text { HOJA }\end{array}$ & $\begin{array}{c}\text { TCR } \\
\text { TALLO }\end{array}$ \\
\hline Tratamiento & 1 & & & & & & & & & & \\
\hline Altura del brote & 0.37 & 1 & & & & & & & & & \\
\hline Ramas & 0.81 & 0.05 & 1 & & & & & & & & \\
\hline Hojas & 0.76 & 0.09 & 0.96 & 1 & & & & & & & \\
\hline$\%$ Materia seca & 0.46 & 0.33 & 0.48 & 0.59 & 1 & & & & & & \\
\hline \% Ceniza & -0.22 & $3.60 \mathrm{E}-03$ & -0.14 & -0.23 & -0.7 & 1 & & & & & \\
\hline$\%$ Proteina & -0.27 & -0.22 & -0.56 & -0.6 & -0.52 & 0.38 & 1 & & & & \\
\hline Peso F. hoja & 0.04 & -0.11 & 0.15 & 0.4 & 0.6 & -0.65 & -0.32 & 1 & & & \\
\hline Peso F. tallo & -0.1 & 0.03 & 0.04 & 0.31 & 0.57 & -0.56 & -0.38 & 0.96 & 1 & & \\
\hline TCR* HOJA & -0.01 & -0.05 & 0.09 & 0.34 & 0.6 & -0.67 & -0.35 & 0.99 & 0.97 & 1 & \\
\hline TCR TALLO & -0.13 & 0.14 & -0.07 & 0.19 & 0.54 & -0.59 & -0.33 & 0.92 & 0.98 & 0.96 & 1 \\
\hline
\end{tabular}

*TCR: Tasa de crecimiento relativo

delgados, con una baja producción de biomasa (Mommer et al., 2006). Esto se debe a que el incremento de temperatura influye en los patrones de precipitación y afecta el desarrollo de los cultivos (Varas y Herrera, 2019). Además, la frecuencia de corte (periodos superiores a los 40 días) permite que la planta acumule reservas y produzca rebrotes más vigorosos (Ojiako et al., 2011). En este caso se encontró que la mayor producción de biomasa se obtuvo en la segunda cosecha y esto pudo deberse al efecto cicatrizante de la planta respecto a la poda inicial, lo cual no sucedió en la segunda cosecha. La producción de biomasa fresca estuvo afectada no solo por la altura de poda, sino también entre la primera y segunda cosecha. Este resultado coincide con lo indicado por Crosby y Craker (2007) quienes mencionan que la biomasa producida como resultado de la primera poda es baja debido a la recuperación del árbol y a la activación de los sistemas de rebrote. Después de la poda, la biomasa fracción gruesa tiende a ser más delgada y numerosa y puede ser utilizada como forraje.

Fonseca et al. (2015) señalan que la altura de poda y la frecuencia de corte tienen efectos crecientes y positivos en moringa, alcanzando los más altos rendimientos a la altura de $0.40 \mathrm{~m}$ (respecto al suelo) y con una frecuencia de corte de 60 días. En este caso el más alto rendimiento promedio obtenido correspondió a la altura de $1.50 \mathrm{~m}$ que fue equivalente a $592.48 \mathrm{Kg} \mathrm{ha}^{-1}$ de biomasa fresca. Aunque la producción de biomasa a diferentes alturas de corte puede variar una a otra cosecha y puede no tener diferencias significativas al analizarse a través del tiempo (Padilla et al., 2014). Zheng et al. (2016) indican que una mayor densidad de siembra $(0.2 \mathrm{~m}$ x $0.2 \mathrm{~m})$ en combinación con una altura de corte de $(0.30 \mathrm{~m})$ produjo el mayor rendimiento de materia fresca (8.43-76.41 t $\mathrm{ha}^{-1}$ ) y seca (1.66-12.85 $\left.\mathrm{t} \mathrm{ha}^{-1}\right)$. Normalmente el desarrollo de moringa está asociado al tipo de clima, suelo, $\mathrm{pH}$ y prácticas de manejo agronómico. Estos factores determinan la altura, el diámetro del tallo y la producción de biomasa (Santiago y Bezerra, 2017). Por ello, al comparar el cultivo moringa

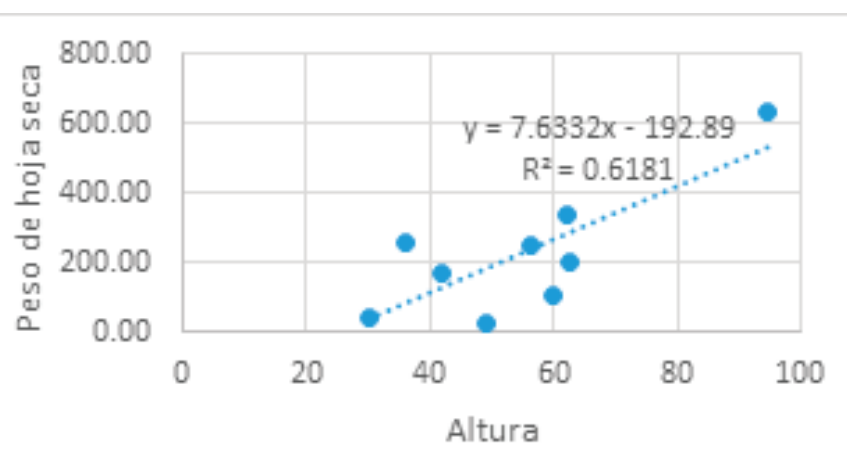

Figura 4. Regresión de la altura de poda con el peso seco de la hoja seca. Figure 4. Regression of pruning height versus dry leaf dry weight.

en diferentes localidades muestran grandes diferencias en los componentes del rendimiento. Moringa puede llegar a alcanzar rendimientos elevados en altas densidades de siembra y a diferentes frecuencias de corte (Mendieta et al., 2013).

\section{Valor bromatológico.}

El contenido proteico en hojas a los 61 y 63 dias entre tratamientos fue similar. Castillo et al. (2013) mencionan que la altura del corte tiene poco efecto en la composición química de las hojas y tallos de moringa. A diferencia de la poda, la frecuencia de corte logra concentrar mayor contenido de proteína encontrándose hasta un $22.8 \%$ de proteína en periodos de cosecha de 75 días (Reyes et al., 2006) y de 23 a 27\% de proteína cruda en hojas en periodos de corte de 100 días (González, 2013). El contenido de proteína en las hojas secas de moringa fue $16.85 \%$. Este valor es mayor que 10.74, 7.12 y $14.40 \%$ reportados por Valdez et al. (2015), Ayasan (2015) y Chucu y Chucu (2013) respectivamente. Sin embargo, este valor es menor que el $27.51 \%$ reportado por Ajantha et al. (2018). Clement et al. (2017) y Hernandez-Torres et al. (2018) mencionan que las condiciones de deshidratado influyen en el contenido sensorial y nutricional (Alvarez-Parrilla et al., 
2019), siendo el deshidratado por radiación directa el más perjudicial en el contenido proteico. El contenido promedio de humedad en la hoja fue del $77.5 \%$ y $17.5 \%$ de proteína superior a lo reportado por Tapia et al. (2012) a una frecuencia de corte de 45 días. Bamishaiye et al. (2011) mencionan que los peridos de cosecha influyen en la acumulación de nutrientes en las hojas. De acuerdo con Nouman et al. (2013), el contenido de proteína cruda, minerales $(\mathrm{K}, \mathrm{Ca}, \mathrm{Mg}$ y $\mathrm{P})$, clorofila, $\beta$-caroteno y contenido fenólico total en las hojas de moringa no se ve influenciado por la altura de poda. La estación de cosecha tiene en efecto en el contenido de proteína. Además, las hojas maduras logran almacenar una mayor cantidad de clorofila en comparacion con las hojas de una planta recien podada (Du Toit et al., 2019). El porcentaje de ceniza en las hojas de moringa fue de $12 \%$, superior a $9.84 \%$ obtenido de un rendimiento de $4.51 \mathrm{t} \mathrm{ha}^{-1}$ de materia seca con cortes de 45, 60 y 75 días (Reyes et al., 2006). Dicho valor se encuentra dentro del rango 6.49 - $14.16 \%$ reportado por Sánchez et al. (2006). El contenido bromatológico en las hojas esta influenciado por la densidad de siembra, periodo de establecimiento, frecuencia de corte, método de secado, variedad genética, fertilidad y tipo de suelo, variaciones ambientales y manejo agronómico (Asante et al., 2014; ValdiviéNavarro et al., 2019).

\section{CONCLUSIONES}

Se concluye que moringa tiene gran tolerancia y responde bien a la poda. Por ello, normalmente se recomienda esta práctica agronómica que permite incrementar la ramificación y, por ende, aumentar la producción de hoja (follaje) y facilita la cosecha. El crecimiento de los brotes permitió una producción aceptable de hojas en periodos de 63 y 61 días, con una mayor producción en la segunda cosecha respecto a la primera después de la poda. Se recomienda realizar podas en moringa a una altura de $1.50 \mathrm{~m}$ para promover la producción de biomasa, a una frecuencia de corte superior a los 45 días para una mayor concentracion de proteína.

\section{REFERENCIAS}

Adedapo, A.A., Mogbojuri, O.M. y Emikpe, B.O. 2009. Safety evaluations of the aqueous extract of the leaves of Moringa oleifera in rats. Journal of Medicinal Plants Research. 3(8): 586-591.

Aguilar-Jiménez, A.N., Magaña-Magaña, M.Á. y CarminiaContreras. L. 2019. Factores socioeconómicos asociados a la diversidad pecuaria del traspatio en comunidades mayas de Yucatán y Campeche. Biotecnia. 21 (3): 5-12.

Ajantha, A., Kathirvelan, C., Purushothaman, M.R. y Visha, P. 2018. Study on Nutrients, Mineral and Vitamin Profile of Moringa oleifera Leaf Meal. International Journal of Current Microbiology and Applied Sciences (IJCMAS). 5: 2478-2481.

Alvarez-Parrilla E., Contreras-Muñiz, F., Rodrigo-García, J., De la Rosa, L.A., García-Fajardo, J.A. y Núñez-Gastélum. J.A. 2019. Cinética de secado y efecto de la temperatura sobre las características físicas y compuestos fenólicos de chile jalapeño rojo (Capsicum annuum I.). Biotecnia. 21 (1): 139147.
Amaglo, N. 2006. How to Produce Moringa Leaves Efficiently?. Kwame Nkrumah University of Science and Technology, Ghana. Rucuperado de http://miracletrees.org/moringadoc/ how_to_produce_moringa_leaves_efficiently.pdf.

Anwar, F., Latif, S., Ashraf, M. y Gilani, A. H. 2007. Moringa oleifera: a food plant with multiple medicinal uses. Phytotherapy Research: An International Journal Devoted to Pharmacological and Toxicological Evaluation of Natural Product Derivatives. 21(1): 17-25.

Asante, W.J., Nasare, I.L., Tom-Dery, D., Ochire-Boadu, K. y Kentil, K.B. 2014. Nutrient composition of Moringa oleifera leaves from two agro ecological zones in Ghana. African Journal of Plant Science. 8(1): 65-71.

Association of Official Agricultural Chemists (AOAC). 1984. Official Methods of Analysis. 15th ed. Association of Official Analytical Chemists. Washington, D.C., USA.

Ayasan, T. 2015. Use of Moringa oleifera in poultry and ruminant nutrition. Turkish Journal of Agriculture-Food Science and Technology. 3(6): 425-429.

Bamishaiye, E.I., Olayemi, F.F., Awagu E.F.y Bamshaiye, O.M. 2011. Proximate and phytochemical composition of Moringa oleifera leaves at three stages of maturation. Advance Journal of Food Science and Technology. 3(4): 233-237.

Bosch, C.H., 2004. Moringa oleifera Lam. record from protabase. In: Grubben, G.J.H, Denton, O.A. (Eds.), PROTA (Plant Resources of Tropical Africa / Resources (Veg etales de L'afrique Tropicale). Wageningen, Netherlands.

Castillo, A., Castillo, C., Ramírez, J.B., Ávilas, L. y Cantos, R. 2013. Efecto de la densidad y frecuencia de la poda en el rendimiento y calidad de la Moringa oleifera Lam. XIII Congreso de la Asociación Latinoamericana de Producción Animal (ALPP) $87 \mathrm{p}$.

Chelliah, R., Ramakrishnan, S. y Antony, U. 2017. Nutritional quality of Moringa oleifera for its bioactivity and antibacterial properties. International Food Research Journal. 24(2): 825833.

Clement, A., Olatunde, M., Patrick, O. y Joyce, O. 2017. Effect of drying temperature on nutritional content of Moringa oleifera leave. World Journal of Food Science and Technology. 1(3): 93-96.

Diario Oficial de la Federación (DOF). 1961. NMX-F-066-S-1978. Determinación de cenizas en alimentos. México.

Du Toit, E.S., Sithole, J. y Vorster, J. 2019. Pruning intensity influences growth, flower and fruit development of Moringa oleifera Lam. under sub-optimal growing conditions in Gauteng, South Africa. South African Journal of Botany. 1(47): 1-9.

Ekhuemelo, D.O. y Udo, A.M. 2016. Investigation of variations in the fibre characteristics of Moringa oleifera (Lam) stem for pulp and paper production. International Journal of Science and Technology 5(1): 19-25.

FAO. 2017. El trabajo de la FAO sobre el cambio climático. Conferencia de las Naciones Unidas sobre el cambio climático 2017. Recuperado de http://www.fao.org/3/ai8037s.pdf

Foidl, N., Mayorga, L., y Vásquez, W. 2011. Utilización del marango (Moringa oleifera) como forraje fresco para ganado. Recuperado de: biomasa@ibw.com.nihttp://www.fao.org/ ag/aga/AGAP/frg/AGROFOR1/Agrofor1.html .

Fonseca, R.F., Molinet, A.S., Santiesteban, R.S., Anaya, K.T. y Torres, M.V. 2015. Efecto de combinaciones de altura y frecuencia 
de corte sobre el rendimiento de la Moringa (Moringa oleifera Lam.) en Granma. Revista Granma Ciencia. 19(2): 1-7.

Gandji, K., Chadare, F.J., Idohou, R., Salako, V.K., Assogbadjo, A.E. y Kakaï, R.G. 2018. Status and utilisation of Moringa oleifera Lam: A review. African Crop Science Journal. 26(1): 137-156.

Hernández-Torres Y., Castillo-Zamudio, R.I., Pérez-Vázquez A. y Salgado-Cervantes M.A. 2018. Efecto del tipo de secador sobre la calidad fisicoquímica de harina de Moringa (Moringa oleifera L.). Investigación y Desarrollo en Ciencia y Tecnología de Alimentos. 3: 423-429.

Isah, A.D., Bello, A.G. y Zarumaye, S.A. 2014. Effects of cutting heights and interval of cutting on the yield of Moringa oleifera (horse raddish). International Journal of Development and Sustainability. 3(5): 1147-1151.

Kakengi, A.M.V., Kaijage J.T., Sarwatt S.V., Mutayoba S.K., Shem M.N. y Fujihara T. 2007. Effect of Moringa oleifera leaf meal as a substitute for sunflower seed meal on performance of laying hens in Tanzania. Livestock Research for Rural Development. 19(8):446.

Lawal, B.A., Olawepo, T.F., Asaolu, V.O., Akanbi, W.B., Jolaoso, M.A. y Ojo, A.M. 2015. Effect of Different Methods of Establishment on Growth and Biomass Yield of Moringa (Moringa oleifera Lam). International Journal of Agriculture and Crop Sciences. 8(4): 650-653.

Ledea, J.L., Roselle, G., Benítez, D., Cruz, J.M., y Arias, R.C. 2018. Sprouting and development of Moringa oleifera Lam. plantlets, established with agamic seed. Cuban Journal of Agricultural Science. 52(1): 1-7.

Leone, A., Spada, A., Battezzati, A., Schiraldi, A., Aristil, J. y Bertoli, S. 2015. Cultivation, genetic, ethnopharmacology, phytochemistry and pharmacology of Moringa oleifera leaves: an overview. International journal of molecular sciences. 16(6): 12791-12835.

Madera-Santana J.T., De Dios-Aguilar, M.A., Colín-Chávez, C., Mariscal-Amaro, L.A., Núñez-Colín, C.A., Veloz-García, R., Guzmán-Maldonado, S.H., Peña-Caballero, V., GrijalvaVerdugo, C.P. y Rodríguez-Núñez, J.R. 2019. Recubrimiento a base de quitosano y extracto acuoso de hoja de Moringa oleifera obtenido por UMAE y su efecto en las propiedades fisicoquímicas de fresa (Fragaria x ananassa). Biotecnia. 14: 33-43.

Mendieta, A.B., Spörndly, E., Reyes, S.N., Salmerón, M.F. y Halling, M. 2013. Biomass production and chemical composition of Moringa oleifera under different planting densities and levels of nitrogen fertilization. Agroforestry Systems. 87(1): 81-92.

Mommer, L., Lenssen, J., Huber, H., Visser, E. y De Kroon, H. 2006. Ecophysiological determinants of plant performance under flooding: a comparative study among seven plant families. Journal of Ecology. 94: 1117-1129.

Montesinos, S. 2010. Moringa oleifera: un árbol promisorio para la ganadería. Asociación Cubana de Producción Animal (acpa). 2: 50-53.

Mudyiwa, S.M., Gadzirayi, C.T., Mupangwa, J.F., Gotosa, J. y Nyamugure, T. 2013. Constraints and opportunities for cultivation of Moringa oleifera in the zimbabwean smallholder growers. International Journal of Agricultural Research, Innovation and Technology. 3(1): 12-19.

Murrieta, M.J.R. 2014. Determinación de la altura óptima de poda del cultivo de moringa (Moringa oleifera) con fines de producción en la zona de Babahoyo. Tesis de grado para obtener el título de Ingeniero Agrónomo. Universidad Técnica de Babahoyo. Ecuador. Recuperado de http://rraae. org.ec/Record/0040_bfb9788e7aa7d4c461859f7e45766877

Narváez, O.J.E. y Moreno, J.C.L. 2005. Evaluación de la producción de forraje de Cnidoscolus aconitifolium (Mill) L.M. Johnst, Moringa oleifera Lam. y Leucaena leucocephala Lam. de Wit, para banco proteico en Pacora, San Francisco Libre, Nicaragua. Ciencia Animal 1: 54-59. Recuperado de http:// repositorio.una.edu.ni/id/eprint/2278

Norma Oficial Mexicana NOM-021-RECNAT-2000. Especificaciones de fertilidad, salinidad y clasificación de suelos. estudios, muestreo y análisis. Disponible en: http://www.ordenjuridico.gob.mx/Documentos/Federal/ wo69255.pdf.

Nouman, W., Siddiqui, M.T., Basra, S.M.A., Farooq, H., Zubair, M. y Gull, T. 2013. Biomass production and nutritional quality of Moringa oleifera as a field crop. Turkish Journal of Agriculture and Forestry. 37(4): 410-419.

Ojiako, F.O., Adikuru, N.C. y Emenyonu, C.A. 2011. Critical issues in Investment, Production and Marketing of Moringa oleifera as an Industrial Agricultural raw material in Nigeria. Journal of Agricultural Research and Development. 10: 39-56.

Olson, M.E. y Fahey, J.W. 2011. Moringa oleifera: un árbol multiusos para las zonas tropicales secas. Revista Mexicana de Biodiversidad, 82(4). 1071-1082.

Orona, I.C., Olivares, E.S., Vázquez, C.V. y Gallegos, M.A.R. 2015. Cultivo de árbol de Moringa (Moringa oleifera) y sus usos potenciales. Memoria de la XXVII Semana Internacional de Agronomía FAZ-UJED. 1: 55-66.

Padilla, C., Fraga, N., Scull, I., Tuero R. y Sarduy, L. 2014. Efecto de la altura de corte en indicadores de la producción de forraje de Moringa oleifera vc. Plain. Revista Cubana de Ciencia Agrícola 48(4): 405-409.

Padilla, C., Valenciaga, N., Crespo, G., González, D. y Rodríguez, I. 2017. Requerimientos agronómicos de Moringa oleifera Lam. en sistemas ganaderos. Livestock Research for Rural Development. 1: 1-20.

Palada, M.C. y Chang, L.C. 2003. Suggested Cultural Practices for Moringa. International Cooperators' Guide AVRDC. AVRDC 1: 03-545.

Ramos, T.O., Castillo, H.J. y Sandoval, G.J.J. 2015. Effect of cutting intervals and heights in forage productivity of Moringa oleifera. Revista Bio Ciencias, 3(3): 187-194.

Reyes, N.S., Ledin, S. y Ledin, I. 2006. Biomass production and chemical composition of Moringa oleifera under different management regimes in Nicaragua. Agroforestry Systems. 66: 231-242.

Rodiles-López, J.O., Arriaga-Martínez, L.P., Martínez-Flores, H.E., Zamora-Vega, R. y García-Martínez, R.M. 2019. Desarrollo de una tortilla adicionada con harinas de aguacate y nopal y su efecto en la reducción de colesterol, triglicéridos y glucosa en ratas. Biotecnia. 21(2): 71-77.

Sánchez, N. R., Spörndly, E. y Ledin, I. 2006. Effect of feeding different levels of foliage of Moringa oleifera to creole dairy cows on intake, digestibility, milk production and composition. Livestock Science. 101(1-3): 24-31.

Santiago, M.T.B. y Bezerra, N.E. 2017. Ecophysiology of Moringa oleifera Lam. in function of different rainfall conditions. Revista Geama. 3(4): 236-241.

Santiesteban, R., Tamayo, E., Verdecia, P., Estrada, J., Diéguez, J., Molinet, D., Espinosa, S., Espinosa, A. y Cordovi, C. 
2012. Influencia de la altura y la frecuencia de corte en el rendimiento de Moringa oleifera. I Taller Nacional de Moringa. Instituto de Ciencia Animal, Cuba.

Sharmila-Bharathi, C., Pugalendhi, L. y Pushpanathan, K.R. 2018. Off season production of annual Moringa (Moringa oliefera Lam.) Cv. PKM 1 through canopy management and chemical manipulation practices. Journal of Pharmacognosy and Phytochemistry. 1: 2218-2224.

Strik, B. y Buller, G. 2005. The impact of early cropping on subsequent growth and yield of highbush blueberry in the establishment years at two planting densities is cultivar dependant. HortScience. 40(7): 1998-2001.

Tapia, D., Borges, J.A., Barrios, M. y León, M. 2012. Fertilización foliar en moringa bajo condiciones de vivero. INIA Divulga. 1: $22-25$.

Valdés, R.O.A., Pérez, V.A., Palacios, W.O.M. y Ruiz, H.R. 2014. Potencial de la asociación Moringa y Ricinus en el subtrópico veracruzano. Revista Mexicana de Ciencias Agrícolas. 9: 1673-1686.
Valdez-Solana, M.A., Mejía-García, V.Y., Téllez-Valencia, A., García-Arenas, G., Salas-Pacheco, J., Alba-Romero, J.J. y Sierra-Campos, E. 2015. Nutritional content and elemental and phytochemical analyses of Moringa oleifera grown in Mexico. Journal of Chemistry. 1: 1-10.

Valdivié-Navarro, M., Martínez-Aguilar, Y., Mesa-Fleitas, O., Botello-León, A., Hurtado, C.B. y Velázquez-Martí, B. 2019. Review of Moringa oleifera as forage meal (leaves plus stems) intended for the feeding of non-ruminant animals. Animal Feed Science and Technology, 114338: 1-9.

Varas, M. C. y Herrera M. S. 2019. Caracterización del período de crecimiento agroclimático del maiz (Zea maíz) en la provincia los Ríos. Biotecnia. 21(1): 54-59

Zheng, Y., Zhang, Y. y Wu, J. 2016. Yield and quality of Moringa oleifera under different planting densities and cutting heights in southwest China. Industrial Crops and Products. 91: 88-96. 